\title{
Colonization of Phlebotomus papatasi changes the effect of pre-immunization with saliva from lack of protection towards protection against experimental challenge with Leishmania major and saliva
}

Sami Ben Hadj Ahmed ${ }^{1,2}$, Belhassen Kaabi ${ }^{3}$, Ifhem Chelbi ${ }^{1}$, Saifeddine Cherni ${ }^{1}$, Mohamed Derbali ${ }^{1}$, Dhafer Laouini ${ }^{4}$ and Elyes Zhioua ${ }^{*}$

\begin{abstract}
Background: Sand fly saliva has been postulated as a potential vaccine or as a vaccine component within multi component vaccine against leishmaniasis. It is important to note that these studies were performed using longterm colonized Phlebotomus papatasi. The effect of sand flies colonization on the outcome of Leishmania infection is reported.

Results: While pre-immunization of mice with salivary gland homogenate (SGH) of long-term colonized (F5 and beyond) female Phlebotomus papatasi induced protection against Leishmania major co-inoculated with the same type of SGH, pre-immunization of mice with SGH of recently colonized (F2 and F3) female P. papatasi did not confer protection against L. major co-inoculated with the same type of SGH. Our data showed for the first time that a shift from lack of protection to protection occurs at the fourth generation (F4) during the colonization process of P. papatasi.

Conclusion: For the development of a sand fly saliva-based vaccine, inferences based on long-term colonized populations of sand flies should be treated with caution as colonization of P. papatasi appears to modulate the outcome of L. major infection from lack of protection to protection.
\end{abstract}

\section{Background}

Leishmaniasis is a neglected tropical disease affecting two million people per year worldwide [1]. Sand flies are the main vector of Leishmania, the etiologic agent of leishmaniasis. Depending on the sand fly and Leishmania species, different clinical forms of the disease from cutaneous, muco-cutaneous, and visceral occur. Control of leishmaniasis is based largely on chemical therapy and vector control measures. However, these methods have met with variable success $[2,3]$. To date no effective vaccine is available [4].

* Correspondence: elyes.zhioua@gmail.com

'Laboratory of Vector Ecology, Institut Pasteur de Tunis, Tunis, Tunisia Full list of author information is available at the end of the article
During blood meals, sand flies salivate into the host's skin. Beyond the functions associated with overcoming vertebrate homeostasis, sand fly saliva modulates the inflammatory response of the host and displays many immunomodulatory properties [5]. Sand fly saliva contains an array of bioactive molecules that allow the vector to successfully obtain a blood meal and enhance transmission of Leishmania promastigotes into a vertebrate host [5]. Among some of the most abundant molecules are anticlotting, antiplatelet, and vasodilatory compounds that increase the hemorrhagic pool where sand flies feed [5].

Sand fly saliva was shown to exacerbate Leishmania infection [6,7]. Several studies reported that pre-immunization with salivary gland homogenates (SGH), salivary 
component, or pre-exposition to uninfected bites of Phlebotomus papatasi provided significant protection against infection with Leishmania major, the etiologic agent of zoonotic cutaneous leishmaniasis (ZCL) [8-10]. All these studies were performed with long-term colonized female $P$. papatasi. Recent data by our group demonstrated that pre-immunization with SGH of wildcaught or with SGH of recently colonized (F1) female $P$. papatasi did not confer protection against $L$. major compared to a significant protection obtained with SGH of long-term colonized ones (F29) [11]. Therefore, during the colonization process, the effect of SGH shifts from lack of protection towards protection. As most studies conducted on sand fly biology rely on colonized sand flies, our principal objective was to determine at which generation this shift from lack of protection to protection against lesion development and parasite load occurs.

\section{Materials and methods}

Sand flies, parasites and animals

Wild sand flies were collected using CDC light traps from an animal shelter located in the village of Felta (governorate of Sidi Bouzid), a highly endemic focus of ZCL [12]. Phlebotomus papatasi was found to be the most abundant sand fly species caught in this area [12]. A new colony of $P$. papatasi (Tunisian strain) was initiated at the Vector Ecology Laboratory of the Institut Pasteur de Tunis [13]. Six generations of P. papatasi (F2, F3, F4, F5, F6 and F14) were used in this study. Since the number of protein components in SGH of $P$. papatasi increases with age and produces a typical electrophoretic pattern within three to five days after emergence [14], sand flies were dissected at three to seven days after emergence. Salivary glands were removed under a stereo microscope in cold phosphate-buffer saline (PBS) $\left(8 \mathrm{mM} \mathrm{Na}_{2} \mathrm{HPO}_{4}, 1.75 \mathrm{mM} \mathrm{KH}_{2} \mathrm{PO}_{4}, 0.25\right.$ $\mathrm{mM} \mathrm{KCl}, 137 \mathrm{mM}),(\mathrm{pH} 7.4)$, and stored in groups of 20 pairs in $20 \mu \mathrm{l}$ of $\mathrm{PBS}$ ( $\mathrm{pH} 7.4$ ) at $-70^{\circ} \mathrm{C}$. Immediately before use, 20 pairs of salivary glands were disrupted in $100 \mu \mathrm{l}$ of PBS buffer by three cycles of freezing-thawing.

A highly virulent strain of L. major MHOM/TN/95/ GLC94, isolated from a Tunisian patient was used in this study [15]. Amastigotes were obtained after passage in $\mathrm{BALB} / \mathrm{c}$ mice in the footpad and harvested from skin lesions by differential centrifugation. Promastigotes were grown on NNN medium at $26^{\circ} \mathrm{C}$ and then progressively adapted to RPMI 1640 medium (Sigma, St Louis, Mo.) containing $2 \mathrm{mM} \mathrm{L}$-glutamine, $100 \mathrm{U}$ of penicillin/ml, $100 \mu \mathrm{g}$ of streptomycin $/ \mathrm{ml}$, and $10 \%$ heat-inactivated foetal calf serum (complete medium). Promastigotes were collected while in the stationary growth phase (enriched metacyclic) by centrifugation $(3000 \times \mathrm{g}, 10$ min, $14^{\circ} \mathrm{C}$ ), washed three times in PBS and re-suspended to the appropriate concentration.
BALB/c mice were bred in the animal facility the Institut Pasteur de Tunis under pathogen-free conditions. Female mice aged between six and eight weeks were used in this study. All experiments involving $\mathrm{BALB} / \mathrm{c}$ mice were performed in accordance with protocols approved by the Institutional Animal Care and Use Committee of Pasteur Institute of Tunis.

\section{Immunizations with SGH and challenge}

Mice were anaesthetized by subcutaneous injection of $200 \mu \mathrm{l}$ of ketamine $(10 \mathrm{mg} / \mathrm{ml})$ (Merial, Lyon, France), and immunized intradermally in the right ear with the equivalent of two pairs of salivary glands in $10 \mu \mathrm{l}$ of PBS. Six groups of 10 mice each were pre-immunized with SGH obtained from sand fly generations F2, F3, F4, F5, F6, and F14, once a week for two weeks. In the fourth week, the groups were challenged with $10^{6} \mathrm{~L}$. major promastigotes co-inoculated with the same type of the SGH used in pre-immunizations. Six control groups of 10 mice each were injected with PBS instead of SGH and challenged with promastigotes co-inoculated with each of the six types of SGH. Female BALB/c mice pre-immunized with SGH and six control groups of mice were challenged with a mixture of two pairs of salivary glands in $10 \mu \mathrm{l}$ of PBS and $10^{6}$ stationary phase L. major promastigotes in $50 \mu \mathrm{l}$ of PBS inoculated subcutaneously in the right hind footpad. The footpad swelling at the site of inoculation was monitored at weekly intervals using a vernier calliper. The lesion size was defined as the increase in the footpad thickness after subtracting the size of the contralateral uninfected footpad. These experiments were repeated three times.

\section{Evaluation of parasite load}

Parasite load was evaluated for all groups of mice. At the seventh week post-infection, three mice per each category (F2, F3, F4, F5, F6, F14, and their respective control groups) were used to determine the parasite load. For each mouse, parasite burden was assessed for the following tissues: footpad lesion, lymph nodes, and spleen. The number of viable parasites present at the site of infection (footpad, draining lymph nodes, and spleen) was quantified using the limiting dilution method [16]. Briefly, each pool of tissue was excised and homogenized in RPMI medium supplemented with $20 \%$ heat-inactivated foetal bovine serum, $100 \mathrm{U}$ of penicillin per $\mathrm{ml}$ and $100 \mu \mathrm{g}$ of streptomycin per $\mathrm{ml}$. Each tissue homogenate was serially diluted in a 96-well Maxisorb plate (Nunc, Roskilde, Denmark). Samples, in quadruplicate, were incubated at $23^{\circ} \mathrm{C}$. The wells containing motile promastigotes were identified under the microscope, and the number of viable parasites in each tissue was determined from the highest dilution at which promastigotes had grown after up to seven days of 
incubation. Results were expressed as the mean $-\log _{10}$ parasite titer.

\section{Statistical analysis}

Using a linear mixed-effects model for longitudinal data, and while allowing for nested random effects (random intercept), and whereas the within-subject residual errors are permitted to be correlated (autoregressive of the first order, AR1) and/or have unequal variances [17], we tested for difference in trends (generation effect) as well as time-generation interaction, between curves illustrating the variation of the lesion size through time for each group of mice immunized and challenged differently as described above. In addition, for specific time point analysis (e.g., post-challenge starting at $3^{\text {rd }}$ week), Wilcoxon [18] and Student's t-test were used to determine median and mean differences in lesion size between groups. Maximum p-value is reported when difference is significant and minimum p-value when it is not. In addition Holm's correction for multiple testing, of the reported $\mathrm{p}$-values was done when appropriate. The test data considered for the analysis consisted of subsets involving different generations combined sequentially starting from F2, and F3 data. The criteria when the model detect a generation effect is a p-value < 0.05 , associated with generation effect. This will show that there is a new generation effect, i.e. a transition from neutral or lack of protection to protective effect. Considering the difference in lesion between groups for post-challenge starting from $3^{\text {rd }}$ week, the same linear mixed model was applied to the obtained difference, to confirm the finding of the above approach. Alternatively, to test for trend or level change i.e. a departure of the mean difference from stationarity, KPSS test was used [19]. To evaluate and test the correlation between parasite load and lesion size, Pearson's statistic was used. All the statistical analyses were performed with the following packages (Stats, Nlme, and Tseries) implemented in the $\mathrm{R}$ software [version 2.10.1] for statistical computing (http://www.r-project.org).

\section{Results}

Footpad lesions of mice pre-immunized with SGH of F5, F6, and F14 female $P$. papatasi developed after challenge with $L$. major co-inoculated with each of the three types of SGH, but they were significantly smaller in size and grew more slowly than in the control groups (max pvalues $<0.0001$ ) (Figures 1, 2). In contrast, mice preimmunized with SGH of F2, F3 female $P$. papatasi challenged with $L$. major co-inoculated with each of the two types of SGH developed lesions as rapidly and as large in size as the control groups $(\mathrm{p}=0.67)$ (Figures 1,2$)$. Mice pre-immunized with SGH of $\mathrm{F} 4$ female $P$. papatasi challenged with $L$. major co-inoculated with the same type of SGH developed lesions less rapidly and less large in size as the control groups, and those pre-immunized with SGH of F2 and F3 female $P$. papatasi, but without significant differences $($ min p-values $=0.1)$. However, lesions size observed in the group of mice pre-immunized with SGH of F4 female $P$. papatasi are larger in size and grow more rapidly than the ones observed in the groups of mice pre-immunized with SGH of F5, F6, and F14 female P. papatasi (p-value < 0.0001) (Figures 1, 2). Lesions size differed significantly in mice pre-immunized with SGH of F2, F3 compared to lesions size observed in the groups of mice pre-immunized with SGH of F5, F6, and F14 female P. papatasi (max p-value $<0.0001$ ).

Considering as test data, subsets involving different generations combined sequentially starting from F2, and F3 data, and the response variable as the difference in lesion size between group pre-immunized with SGH of $\mathrm{Fi}$ and its control group, a generation effect is detected at F4 ( $\mathrm{p}$-value $=0.019)$. This showed that there is a transition from lack of protection to protection. This protective effect phenomenon became clearer starting from the F5 ( $\max$ p-values < 0.001) (Figure 3). Alternatively, the KPSS test for trend and level change indicated a level change, i.e. a departure of the mean difference from stationarity ( $\mathrm{p}$-value $=0.036$ ) (Figure 3 ).

The lesion sizes among different groups were proportionally correlated with parasite burdens in different organs i.e. footpad, lymph nodes, and spleen; $(\min r=$ 0.88 , max $\mathrm{p}<0.001$ ) (Figure 4). A statistically significant difference (max adjusted-p $<0.001$ ) in mean parasite load in the infected footpad of up to $4 \log _{10}$ units was observed in mice pre-immunized with SGH of F5, F6, F14 compared to their control groups respectively, and to those pre-immunized with SGH of F2 and F3 female P. papatasi. A significant difference (max adjusted-p < 0.05 ) in mean parasite load in draining lymph nodes of up to $3 \log _{10}$ units was observed in mice pre-immunized with SGH of F5, F6, F14 compared to their control groups respectively, and to those pre-immunized with SGH of F2 and F3 female P. papatasi. Similarly, a significant difference (max adjusted-p $<0.01$ ) in mean parasite load in the spleen of up to $4 \log _{10}$ units was observed in mice pre-immunized with SGH of F5, F6, F14 compared to their control groups, and to those preimmunized with SGH of F2 and F3 female P. papatasi. The parasite loads in infected footpad, lymph nodes, and spleen were significantly lower in mice pre-immunized with SGH of F5, F6, and F14 than in the group of mice pre-immunized with SGH of F4 female $P$. papatasi (max adjusted $\mathrm{p}<0.05$ ). There is a significant difference in parasite load observed in mice pre-immunized with SGH of F4 compared to the groups of mice pre-immunized with SGH of F2 female $P$. papatasi. The difference in parasite load observed between mice pre-immunized 


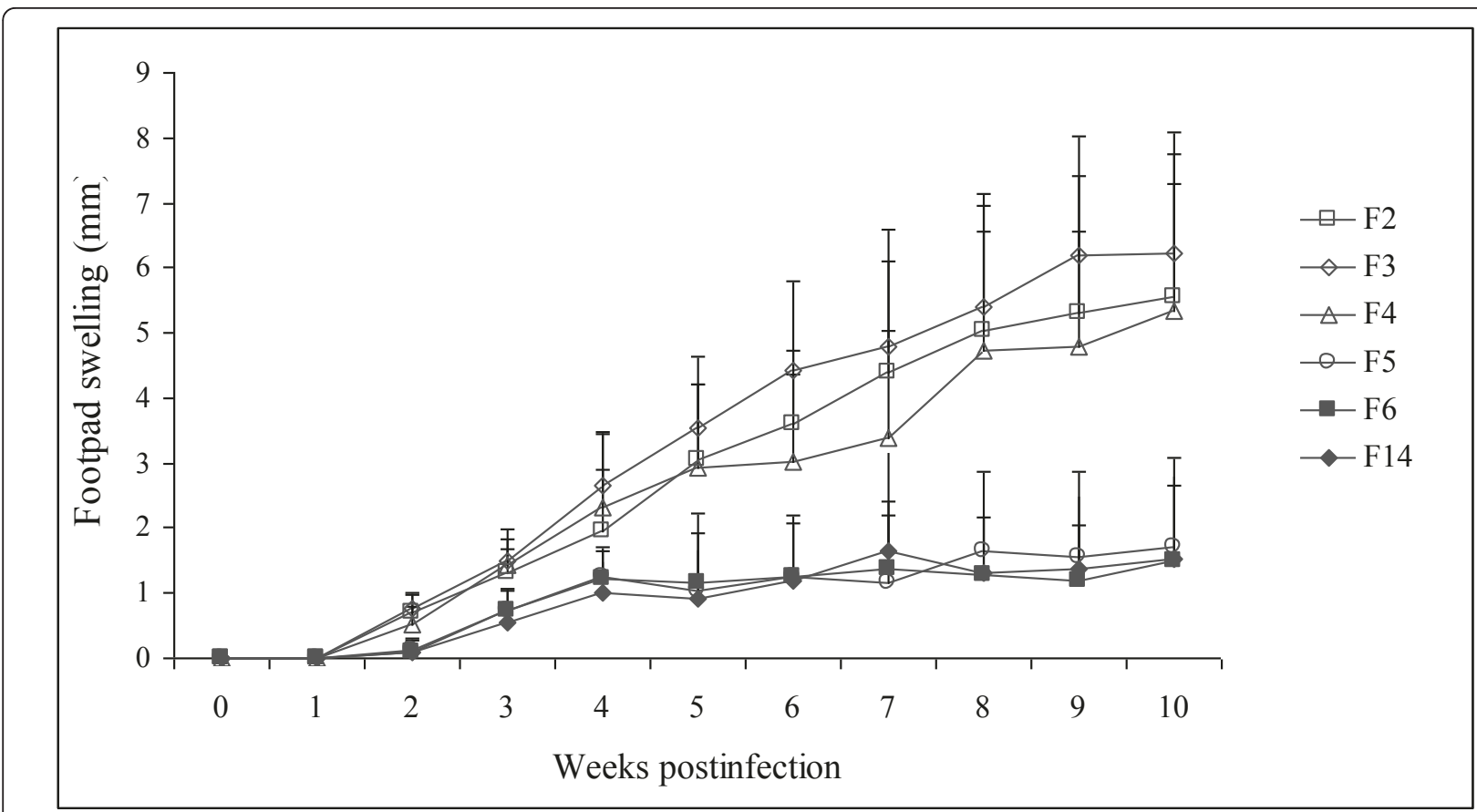

Figure 1 Lesion progression in vaccinated BALB/c mice after challenge with $10^{6}$ L. major metacyclic enriched promastigotes. Each experiment is repeated three times. Results of the 3 experiments (10 mice per group) pooled together are expressed as increases in footpad thickness (in millimetres) and are means + S.D. F2: mice pre-immunized with SGH of female P. papatasi (F2) and challenged with L. major coinoculated with the same type of SGH; F3: mice pre-immunized with SGH of female P. papatasi (F3) and challenged with L. major co-inoculated with the same type of SGH; F4: mice pre-immunized with SGH of female P. papatasi (F4) and challenged with L. major co-inoculated with the same type of SGH; F5: mice pre-immunized with SGH of female $P$. papatasi (F5) and challenged with L. major co-inoculated with the same type of SGH; F6: mice pre-immunized with SGH of female P. papatasi (F6) and challenged with L. major co-inoculated with the same type of SGH; F14: mice pre-immunized with SGH of female P. papatasi (F14) and challenged with L. major co-inoculated with the same type of SGH;

with SGH of F4 and to those pre-immunized with SGH of F3 female $P$. papatasi is not statistically different for the footpad and lymph node but it is statistically significant for the spleen $(\mathrm{p}<0.05)$. No significant difference in parasite load was observed between mice pre-immunized with SGH of F2 and F3 and the control groups ( $\mathrm{p}$ $>0.05$ ) for all tissues tested (Figure 4).

\section{Discussion}

Our results demonstrate that pre-immunization with SGH of F2, and F3 generations of female P. papatasi does not confer protection against L. major. Similarly, pre-immunization with SGH of wild-caught or recently colonized $P$. papatasi (F1) do not confer protection against L. major infection [11]. The shift from lack of protection to protection occurs at the $\mathrm{F} 4$ generation and the protection effect of SGH is first observed at F5 and in following generations. This shift occurring at the F4 generation was within this particular study and the timing is likely to vary from case to case depending on the selection applied and ease/difficulty of adaptation of sand flies from the wild to a laboratory colony. As far as we are aware, this is the first report on the effect of colonization on the outcome of pre-immunization with SGH on L. major infection.

Previous studies showed that pre-immunization with SGH, salivary component, or pre-exposure to uninfected bites of long-term laboratory-colonized female P. papatasi, induced significant protection against $L$. major coinoculated with the same type of SGH [8-10]. These studies were performed with long-term colonized $P$. papatasi. We showed that pre-immunization with SGH of recently colonized $P$. papatasi did not provide protection against $L$. major co-inoculated with the same type SGH compared to a significant protection obtained with SGH of long-term colonized one [11]. The protective effect against $L$. major following pre-exposure of mice twice to uninfected bites of long-term colonized Phlebotomus dubosqi shortly before experimental challenge provided protection against L. major [20]. However, there is a lack of protection when mice are pre-exposed for long-term to uninfected bites of $P$. dubosqi followed by free sand fly bites period prior to infection [20]. It was reported that pre-immunization of BALB/c with SGH of colonized Lutzomyia intermedia did not protect BALB/c mice against Leishmania braziliensis co- 


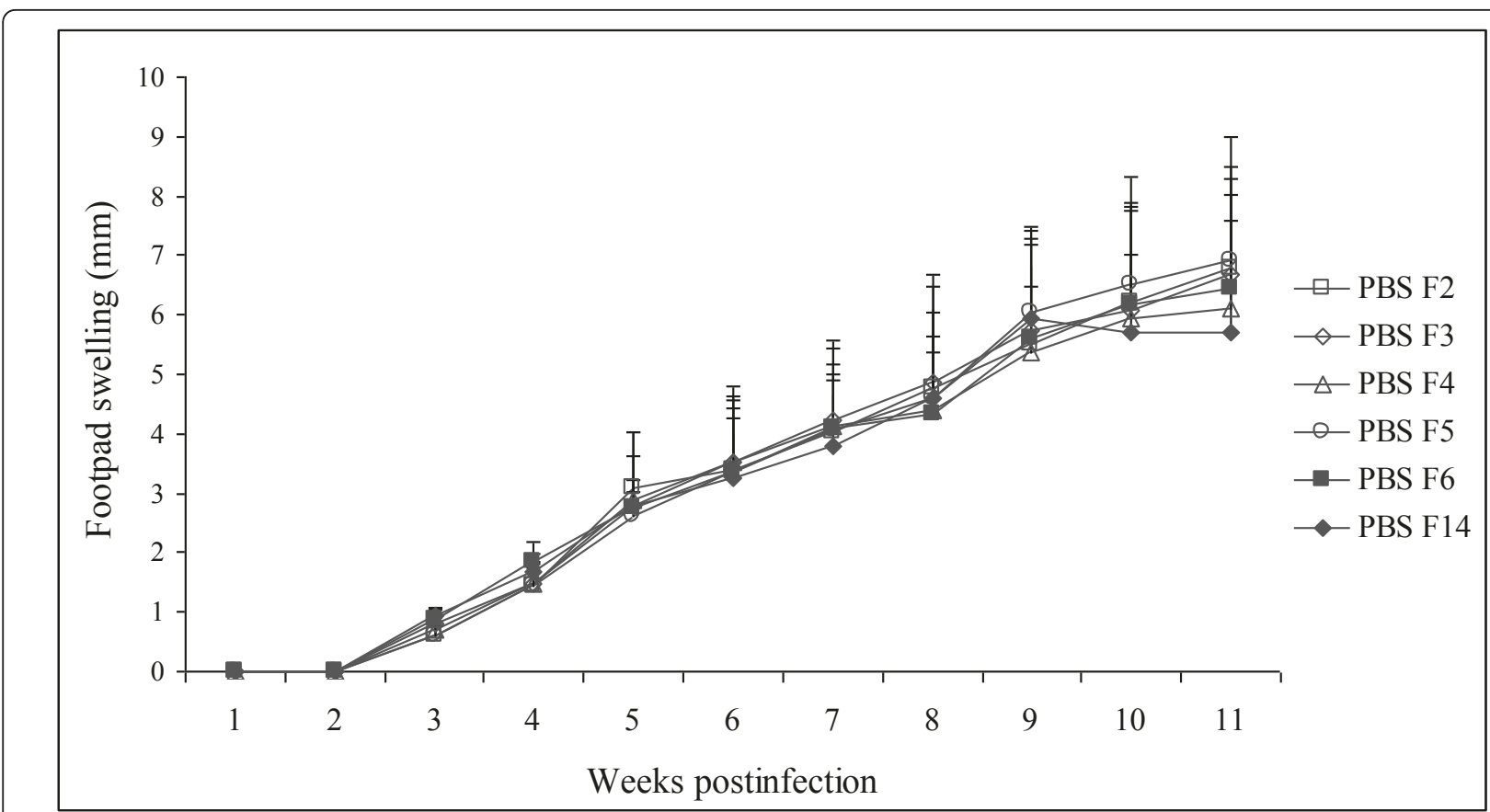

Figure 2 Lesion progression in control BALB/C mice after challenge with $10^{6} \mathrm{~L}$. major metacyclic enriched promastigotes. Each experiment is repeated three times. Results of the 3 experiments (10 mice per group) pooled together are expressed as increases in footpad thickness (in millimetres) and are means + S.D. PBS-F2 (control group): mice pre-immunized with PBS only and challenged with L. major coinoculated with SGH of female P. papatasi (F2); PBS-F3 (control group): mice pre-immunized with PBS only and challenged with L. major coinoculated with SGH of female P. papatasi (F3); PBS-F4 (control group): mice pre-immunized with PBS only and challenged with L. major coinoculated with SGH of female P. papatasi (F4); PBS-F5 (control group): mice pre-immunized with PBS only and challenged with L. major coinoculated with SGH of female P. papatasi (F5); PBS-F6 (control group): mice pre-immunized with PBS only and challenged with $L$. major coinoculated with SGH of female P. papatasi (F6); PBS-F14 (control group): mice pre-immunized with PBS only and challenged with L. major coinoculated with SGH of female P. papatasi (F14);

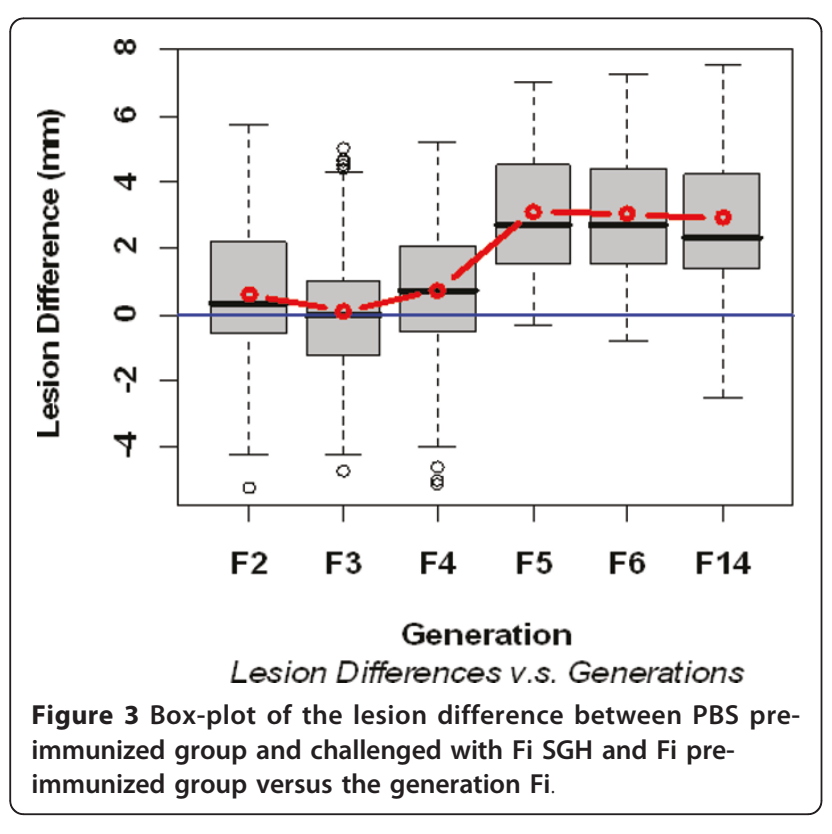

inoculated with the same type of SGH [21]. The use of SGH may represent a hurdle in the development of vaccines based on sand fly saliva and therefore, it is highly needed to identify and select individual salivary protein candidates instead of using SGH [21]. It is important to point out that these authors emphasized that colonization of Lu. intermedia is a challenging process [21]. Thus, the enhancement observed for L. braziliensis infection following pre-exposure of mice to $\mathrm{Lu}$. intermedia saliva was potentially due to the sand fly generation used which was not indicated in their study.

As for ticks, it is well established that there are homologues and prologues in their salivary gland trasncriptomes that likely encode products to circumvent host immune responses that could neutralize their biological activity [22]. Natural selection may occur in natural field populations of sand flies that favor polymorphism of salivary gland proteins and subsequently induce antigenic variation to avoid effects of the host immune system $[23,24]$. This hypothesis is corroborated by our 


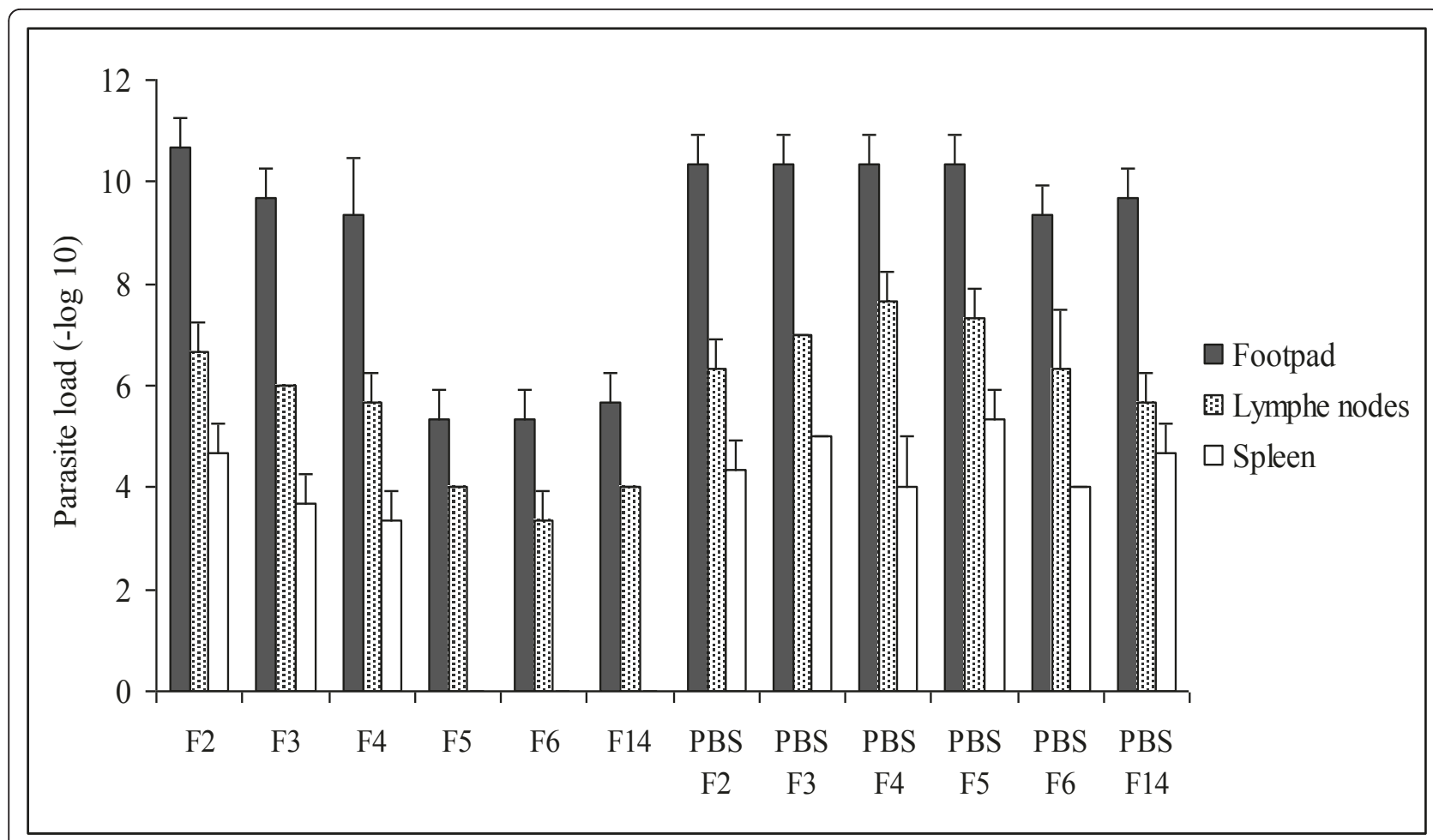

Figure 4 Parasite loads. At seven weeks post-infection, the number of viable parasites was determined from footpad, draining lymph nodes, and spleen of mice pre-immunized with SGH of F2, F3, F4, F5, F6, F14, and control groups. The parasite burdens in the infected tissues were assessed by limiting dilution, as described in Material and Methods. Presented values are the mean +S.D. of three mice per category.

findings showing: 1) that pre-immunization with SGH of wild-caught or recently colonized (F1) P. papatasi do not confer protection against L. major compared to a significant protection obtained with long-term colonized ones, [11] and 2) pre-immunization with SGH of longterm colonized (F39) P. papatasi do not confer protection against $L$. major co-inoculated with SGH of wildcaught compared to a significant protection obtained when both pre-immunization and challenge were performed with SGH of long-term colonized P. papatasi [25]. It was also reported that colonized and wild-caught Lutzomyia longipalpis differ in the composition and the amount of salivary proteins and these differences may account for the lower effect observed on the modulation of experimental Leishmania infection by wild-caught SGH $[26,27]$.

Despite that fact that no direct evidence is presented in this study for a loss of variability in salivary protein genes, we hypothesized that a loss of genetic variation as a result of colonization is potentially responsible for the protection observed in mice pre-immunized with long-term colonized P. papatasi. Conversely, the antigenic diversity of salivary gland proteins of recently colonized $P$. papatasi is likely the reason for lack of protection in mice pre-immunized with SGH of recently colonized flies.
SP-15 was shown to be protective against $L$. major [10]. It was hypothesized that the development of a vaccine based on SP-15 will not be affected by an inconsistent immune response due to genetic variation in natural populations of $P$. papatasi [28]. However, several studies emphasized that natural genetic variation in candidate salivary vaccines is an important issue in the potential efficacy of a vaccine [11,23-25].

Laboratory colonies of insects are often accepted as being representative of field populations from which they have been derived. However, this assumption may not always be valid, as colonies frequently incorporate only a fraction of the original population's genetic variability [29]. Long-term term colonization of P. papatasi induced a selection of refractory and susceptible lines to L. major [30,31]. Wild-caught $P$. papatasi exhibited the highest genetic variation in SP-15 compared to colonized flies of the same species [28]. Moreover, the analysis of genetic variation at 17 enzyme loci of one colonized and five field populations of $P$. papatasi showed that polymorphism of the examined loci observed in colonized and in field populations were $23.5 \%$ and $76.6 \%$, respectively [32].

For the New World sand fly Lu. longipalpis, colonization led to reduced genetic variability in comparison to field samples, and to fixation of rare or previously 
undetected alleles [33]. Hence, colonization of sand flies may reduce genetic variability and may select for certain traits not present in field populations.

For $P$. papatasi, pre-immunization of mice to PpSP15 was shown to be protective against $L$. major, while immunization with another salivary gland protein PpSP44 from the same colony of $P$. papatasi induced disease enhancement [34]. In addition, pre-immunization with SGH of wild-caught or recently colonized $P$. papatasi does not protect against $L$. major infection contrasting with significant protection observed with long-term colonized flies [11]. Our previous studies revealed that pre-immunization with SGH of long-term colonized (F39) P. papatasi do not confer protection against $L$. major co-inoculated with $\mathrm{SGH}$ of wild-caught compared to a significant protection obtained when both pre-immunization and challenge were performed with SGH of long-term colonized P. papatasi [25].

In conclusions, we provide in this study further evidence that colonization has a direct impact on the outcome of L. major infection following pre-immunization with SGH of different generation of P. papatasi. Moreover, the change in the resulting effect was detected between the $4^{\text {th }}$ and $5^{\text {th }}$ generations following colonization. As indicated above, whether this is related to a qualitative or quantitative difference in the sand fly saliva remains to be determined.

\section{Acknowledgements}

The authors thank Howard Ginsberg, Rhayza Maignon, and Marcelo Ortigao for reviewing the manuscript. The study was part of the post-doctoral research program of Sami Ben Hadj Ahmed.

\section{Author details \\ ${ }^{1}$ Laboratory of Vector Ecology, Institut Pasteur de Tunis, Tunis, Tunisia. ${ }^{2}$ Department of Biology, University of Gafsa, Gafsa, Tunisia. ${ }^{3}$ Laboratory of Epidemiology and Ecology of Parasites, Institut Pasteur de Tunis, Tunis, Tunisia. ${ }^{4}$ Laboratory of Immuno-Pathology, Vaccinology, and Molecular Genetics, Institut Pasteur de Tunis, Tunis, Tunisia.}

\section{Authors' contributions}

SBM carried out the experiments, BK, performed the statistical analysis, IC, SC, MD are maintaining the colony of sand fly and salivary gland dissections, DL provided L. major isolates used in this study, EZ designed the experiment and drafted the first version of the manuscript and finalized the manuscript. All authors read and approved the final version of the manuscript.

\section{Competing interests}

The authors declare that they have no competing interests.

Received: 6 May 2011 Accepted: 4 July 2011 Published: 4 July 2011

\section{References}

1. Desjeux P: Leishmaniasis: current situation and perspectives. Comp Immunol Microbiol Infec 2004, 27:305-318.

2. Alvar J, Yactayo S, Bern C: Leishmaniasis and poverty. Trends Parasitol 2006, 22:552-557.

3. Alexander B, Maroli M: Control of phlebotomine sandflies. Med Vet Entomol 2003, 17:1-18.

4. Handman E: Leishmaniasis: current status of vaccine development. Clin Microbiol Rev 2000, 14:229-243.
5. Ribeiro JM: Role of saliva in blood-feeding by arthropods. Ann Rev Entomol 1987, 32:463-478.

6. Titus R, Riberiro JMC: Salivary gland lysates from the sand fly Lutzomyia longipalpis enhance Leishmania infectivity. Science 1988, 239:1306-1308.

7. Mbow ML, Bleyenberg JA, Hall LR, Titus R: Phlebotomus papatasi sand fly salivary gland lysate down-regulates a Th1, but up-regulates a Th2, response in mice infected with Leishmania major. J Immunol 1998, 161:5571-5577.

8. Belkaid Y, Kamhawi S, Modi G, Valenzuela J, Noben-Trauth N, Rowton E, Ribeiro J, Sacks DL: Development of a natural model of cutaneous leishmaniasis: powerful effects of vector saliva and saliva pre-exposure on the long-term outcome of Leishmania major infection in the mouse ear dermis. J Exp Med 1998, 188:1941-1953.

9. Kamhawi S, Belkaid Y, Modi G, Rowton E, Sacks D: Protection against cutaneous leishmaniasis resulting from bites of uninfected sand flies. Science 2000, 290:1351-1354.

10. Valenzuela J, Belkaid Y, Garfiela MK, Mendez S, Kamhawi S, Rowton E, Sacks D, Ribeiro JMC: Toward a defined anti-Leishmania vaccine targeting vector antigens: characterization of a protective salivary protein. $J$ Exp Med 2001, 194:331-342.

11. Ben Hadj Ahmed S, Chelbi I, Kaabi B, Cherni S, Derbali M, Zhioua E: Differences in the salivary effects of wild-caught versus colonized Phlebotomus papatasi (Diptera: Psychodidae) on the development of Zoonotic Cutaneous Leishmaniasis in BALB/c Mice. J Med Entomol 2010, 47:74-79.

12. Chelbi I, Derbali M, AL-Ahmadi Z, Zaafouri B, El Fahem A, Zhioua E: Phenology of Phlebotomus papatasi (Diptera: Psychodidae) relative to the seasonal prevalence of zoonotic cutaneous leishmaniasis in Central Tunisia. J Med Entomol 2007, 44:385-388.

13. Chelbi I, Zhioua E: Biology of Phlebotomus papatasi (Diptera: Psychodidae) in the laboratory. J Med Entomol 2007, 44:597-600.

14. Volf $P$, Tesarova $P$, Nohynkova E: Salivary proteins and glycoproteins in phlebotomine sandflies of various species, sex and age. Med Vet Entomol 2000, 14:251-256.

15. Ben Hadj Ahmed S, Bahloul C, Robbana C, Askri S, Dellagi K: A comparative evaluation of different DNA vaccine candidates against experimental murine leishmaniasis due to L. Major. Vaccine 2004, 22:1631-1639.

16. Buffer PA, Sulahian A, Garin YG, Nassar N, Derouin F: Culture microtitration: a sensitive method for quantifying Leishmania major in tissues of infected mice. Antimicrob Agents Chemother 1995, 39:2167-2168.

17. Laird NM, Ware JH: Random-effect models for longitudinal data. Biometrics 1982, 38:963-974.

18. Bauer DF: Constructing confidence sets using rank statistics. J Amer Stat Ass 1972, 67:687-690.

19. Kwiatkowski $D$, Phillips PCB, Schmidt $P$, Shin $Y$ : Testing the null hypothesis of stationarity against the alternative of a unit root. J Econom 1992, 54:159-178.

20. Rohousova I, Hostomoska J, Vlokva M, Kobets T, Lipoldova M, Volf P: The protective effect against Leishmania infection conferred by sand fly bites is limited to short-term exposure. Inter J Parasitol 2011, 41:481-485.

21. de Moura TR, Olivera F, Novais FO, Miranda JC, Follador I, Carvalho EM, Valenzuela JG, Barral-Netoo M, Barral A, Brodskyn C, de Oliveira Cl: Enhanced Leishmania braziliensis infection following pre-exposure to sandfly saliva. PloS Negl Trop Dis 2007, 1:e84.

22. Chmelar J, Anderson JM, Mu J, Jochim RC, Valenzuela JG, Kopecky J: Insight into the sialome of the castor bean tick, Ixodes ricinus. BMC Genomics 2008, 9:233.

23. Lanzaro GC, Lopes AHCS, Riberiro JMC, Shoemaker CB, Warburg A, Soare Ms, G Titus RG: Variation in the salivary peptide, maxadilan from species in the Lutzomyia longipalpis complex. Insect Mol Biol 1999, 8:267-275.

24. Milleron RS, Mutebi JP, Valle S, Montoya A, Yin H, Soong L, Lanzaro GC: Antigenic diversity in maxadilan, a salivary protein from the sand fly vector of American visceral leishmaniasis. Am J Trop Med Hyg 2004, 70:278-283.

25. Ben Hadj Ahmed S, Kaabi B, Chelbi I, Cherni S, Derbali M, Laouini D, Zhioua E: Lack of protection of pre-immunization with saliva of longterm colonized Phlebotomus papatasi against experimental challenge with Leishmania major and saliva of wild-caught P. papatasi. Am J Trop Med Hyg 2010, 83:512-514.

26. Laurenti MD, Silveira VM, Secundino NF, Corbett CE, Pimenta PP: Saliva of laboratory-reared Lutzomyia longipalpis exacerbates Leishmania 
(leishmania) amazonensis infection more potently than saliva of wildcaught Lutzomyia longipalpis. Parasitol Inter 2009, 58:220-226.

27. Laurenti MD, da Matta VL, Pernichelli T, Secundino NF, Pinto LC, Corbett CE, Pimenta PP: Effects of saliva gland homogenate from wild-caught and laboratory-reared Lutzomyia longipalpis on the evolution and immunomodulation of Leishmania (leishmania) amazonensis infection. Scand I Immunol 2009, 70:389-395.

28. Elnaiem DEA, Meneses C, Slotman M, Lanzaro GC: Genetic variation in the sand fly salivary protein, SP-15, a potential vaccine candidate against Leishmania major. Insect Mol Biol 2005, 14:145-150.

29. Lorenz L, Beaty BJ, Aitken THG, Wallis GP, Tabachnik WJ: The effect of colonization upon Aedes aegypti susceptibility to oral infection with yellow fever virus. Am J Trop Med Hyg 1984, 33:690-694.

30. Wu WK, Tesh RB: Selection of Phlebotomus papatasi (Diptera: Psychodidae) line susceptible and refractory to Leishmania major infection. Am J Trop Med Hyg 1990, 42:320-328.

31. Wu WK, Tesh RB: Genetic factors controlling susceptibility and refractoriness to Leishmania major infection in the sand fly Phlebotomus papatasi (Diptera: Psychodidae). Am J Trop Med Hyg 1990, 42:329-334.

32. Kassem HA, Fryauff DJ, Shehata MG, Sawaf BM: Enzyme polymorphism and genetic variability of one colonized and several field populations of Phlebotomus papatasi (Diptera: Psychodidae). J Med Entomol 1993, 30:407-413.

33. Mukhopadhyay J, Rangel EF, Ghosh K, Munstermann L: Patterns of genetic variability in colonized strains of Lutzomyia longipalplis (Dipter: Psychodidae) and its consequences. Am J Trop Med Hyg 1997, 57:216-221.

34. Oliveira F, Lawyer PG, Kamhawi S, Valenzuela JG: Immunity to distinct sand fly salivary proteins primes the anti-Leishmania immune response towards protection or exacerbation of disease. PLOS Neg Trop Dis 2008, 2: e226.

doi:10.1186/1756-3305-4-126

Cite this article as: Ben Hadj Ahmed et al: Colonization of Phlebotomus papatasi changes the effect of pre-immunization with saliva from lack of protection towards protection against experimental challenge with Leishmania major and saliva. Parasites \& Vectors 2011 4:126.

\section{Submit your next manuscript to BioMed Central and take full advantage of:}

- Convenient online submission

- Thorough peer review

- No space constraints or color figure charges

- Immediate publication on acceptance

- Inclusion in PubMed, CAS, Scopus and Google Scholar

- Research which is freely available for redistribution

Submit your manuscript at www.biomedcentral.com/submit
Biomed Central 